\title{
The conceptual framework of the International Tobacco Control (ITC) Policy Evaluation Project
}

\author{
G T Fong, K M Cummings, R Borland, G Hastings, A Hyland, G A Giovino, D Hammond, \\ ME Thompson
}

Tobacco Control 2006;15(Suppl III):iii3-iii1 1. doi: 10.1136/tc.2005.015438

See end of article for authors' affiliations

Correspondence to

Correspondence to:
Geoffrey T Fong, PhD Department of Psychology, University of Waterloo, 200 University Avenue West, Waterloo, Ontario N2L 3G1, Canada; gfong@uwaterloo.ca

Received 12 December 2005

Accepted 26 April 2006
This paper describes the conceptual model that underlies the International Tobacco Control Policy Evaluation Project (ITC Project), whose mission is to measure the psychosocial and behavioural impact of key policies of the Framework Convention on Tobacco Control (FCTC) among adult smokers, and in some countries, among adult non-smokers and among youth. The evaluation framework utilises multiple country controls, a longitudinal design, and a pre-specified, theory-driven conceptual model to test hypotheses about the anticipated effects of specific policies. The ITC Project consists of parallel prospective cohort surveys of representative samples of adult smokers currently in nine countries (inhabited by over $45 \%$ of the world's smokers), with other countries being added in the future. Collectively, the ITC Surveys constitute the first-ever international cohort study of tobacco use. The conceptual model of the ITC Project draws on the psychosocial and health communication literature and assumes that tobacco control policies influence tobacco related behaviours through a causal chain of psychological events, with some variables more closely related to the policy itself (policy-specific variables) and other variables that are more downstream from the policy, which have been identified by health behaviour and social psychological theories as being important causal precursors of behaviour (psychosocial mediators). We discuss the objectives of the ITC Project and its potential for building the evidence base for the FCTC.
$\mathrm{T}$ he global effort to reduce the burden of tobacco use, which is projected to result in the premature deaths of over one billion people in the 21 st century, over $70 \%$ of which will occur in low and middle income countries, culminated in the creation and adoption of the Framework Convention on Tobacco Control (FCTC). ${ }^{12}$ The FCTC is the first-ever international public health treaty. It obligates ratifying countries to broad, comprehensive tobacco control policies. ${ }^{2}$ The main policies of the FCTC are presented in table 1.

The progression of the FCTC has been rapid. One hundred and sixty-eight countries signed the FCTC and the ratification process, which started in May 2003, required a mere 18 months to meet the 40-country threshold for the treaty to come into force. As of April 2006, 126 countries have ratified the FCTC. ${ }^{3}$ The process of setting the specific terms and the governance of the FCTC began in February 2006 at the First Conference of the Parties.

The FCTC has propelled tobacco control into a new era as countries all over the world consider incorporating FCTC policies and recommendations into their own laws. This is a time of great promise and great challenge. As tobacco control policies are formulated and implemented, it is important that policies undergo rigorous evaluation. As intuitively appealing as it may be to put graphic photographs on warning labels, or to restrict sponsorship activities of tobacco companies, these policies must be evaluated to provide concrete evidence of their effects. Such research both serves to evaluate existing policies, and to inform and support future policies. In the same way that evidence-based medicine has been built from rigorous evaluation of treatment options, evidence-based public health policy must begin with building a database from rigorous evaluation of public health policies. Doing so will provide policymakers with the evidence base necessary to implement and defend effective policies, to choose which level of implementation of an FCTC policy may be most desirable (for example, the minimal implementation for warning labels of $30 \%$ of the package versus the recommended 50\%, with graphic elements), and to identify areas where improvement in policy impact might be achieved.

This article describes the International Tobacco Control Policy Evaluation Project (the ITC Project). We describe the challenges of evaluating the national-level policies of the FCTC, the design considerations we weighed in creating our methods, and the conceptual model developed to guide our research on how and why different policies exert their effects on tobacco use behaviours. Other articles in this supplement illustrate the application of the conceptual model in measuring policy effects on tobacco use behaviours and the psychosocial precursors of such behaviours.

\section{EXISTING EVALUATION STUDIES OF TOBACCO CONTROL POLICIES}

Evaluation of tobacco control policies at the population level is in its early stages of development, and as such, studies on the effectiveness of tobacco control policies suffer from design limitations. Cross-sectional studies, for example, are low in internal validity-that is, they are generally weak in their ability to yield causal attributions. ${ }^{4}$ Longitudinal studies are, of course, higher in internal validity, but the limited number of such studies in tobacco policy research often lack comparison groups, and are thus unable to disentangle policy effects from secular trends and historical event threats to internal validity. ${ }^{4-6}$ Furthermore, the few longitudinal studies with comparison communities have focused on local and/or state policies, not national ones. ${ }^{78}$ For example, many US studies have examined differences in smoking behaviour by comparing states with high levels of per capita spending on tobacco control

Abbreviations: ETS, environmental tobacco smoke; FCTC, Framework Convention on Tobacco Control; GYTS, Global Youth Tobacco Survey; ITC, International Tobacco Control, NIH, US National Institutes of Health; SES, socioeconomic status; TTURC, Transdisciplinary Tobacco Use Research Center 
Table 1 Key policy provisions of the Framework Convention on Tobacco Control (FCTC)

- Increase tobacco taxes

- Protect citizens from exposure to tobacco smoke in workplaces, public transport and indoor public places

- Enact comprehensive bans on tobacco advertising, promotion and sponsorship

- Regulate the packaging and labelling of tobacco products to prevent the use of misleading and deceptive terms such as "light" and "mild"

- Regulate the packaging and labelling of tobacco products to ensure appropriate product warnings are communicated to consumers, for example, obligate the placement of rotating health warnings on tobacco packaging that cover at least $30 \%$ (but ideally $50 \%$ or more) of the principal display areas and can include pictures or pictograms

- Regulate the testing and disclosure of the content and emissions of tobacco products

- Promote public awareness of tobacco control issues by ensuring broad access to effective comprehensive educational and public awareness programmes on the health risks of tobacco and exposure to tobacco smoke

- Promote and implement effective programmes aimed at promoting the cessation of tobacco use

- Combat smuggling, including the placing of final destination markings on packs

- Implement legislation and programmes to prohibit the sale of tobacco products to minors

- Implement policies to support economically viable alternative sources of income for tobacco workers, growers, and individual sellers

(COMMIT, California, Florida, Massachusetts) with other states whose tobacco control programmes have not been so well-funded..$^{9-11}$ Sub-national activity inevitably excludes many crucial policies: health warning labels (and other aspects of tobacco packaging), restrictions on "light" or "mild" descriptors, and advertising and marketing restrictions ${ }^{12}{ }^{13}$ are typically implemented at a national or international level. This greater reach increases the potential impact of the policies. ${ }^{14}$ Whatever the value of subnational policies-and in many cases, this value has been substantial-the FCTC has placed national-level policies on centre stage.

It follows from first principles of evaluation design that evaluation of a policy implemented in one country is most rigorous if conducted with reference to one or more other countries in which that policy has not been implemented. In other words, evaluation of FCTC policies is best conducted via international studies. However, there are few international studies of tobacco use. To our knowledge, the only existing international research effort that has some potential in evaluating the impact of FCTC policies is the Global Youth Tobacco Survey (GYTS), which involves school-based surveys of youth in over 150 countries. ${ }^{15}$ Despite its wide scope, however, the GYTS is limited by its cross-sectional design (although the ongoing effort to repeat the GYTS in a large number of countries will increase its value as an evaluation tool), its exclusive focus on youth, and by the limited number of survey items that directly relate to FCTC policies.

Although there exist national surveillance surveys in a number of countries, differences across countries in research methods, questions employed, and the frequency and timing of survey waves reduce their utility for making comparisons. Furthermore, virtually all of the existing national surveillance efforts are crosssectional, which limits the kinds of causal inferences that can be made about the impact of policies on individuals, and thus precludes any specific tests concerning how a given policy operates to affect the behaviour of individuals.

\section{THE CHALLENGE OF EVALUATING TOBACCO CONTROL POLICIES}

It is not possible to conduct randomised experimental studies to evaluate the effects of tobacco control policies because governments, not researchers, control policy implementation. ${ }^{3}$ Furthermore, policy options are often interrelated, so it is often impossible to consider policies in isolation of other events. However, in the absence of a randomised clinical trial, there are four strategies that researchers can employ for the rigorous evaluation of the effects of policies. First is the use of a quasi-experimental design (the so-called "natural experiment") in which one group that is exposed to a policy is compared to a comparable group that is not exposed, as discussed above. Second is the use of a longitudinal cohort design in which individuals are measured on the same key outcome variables at multiple times, preferably before and after a policy is implemented. Combining these two strategies in a single study yields a two-group, pre-post design, which offers a higher degree of internal validity than either feature alone. ${ }^{3}$ The utility of longitudinal designs is strengthened if there are multiple data collections before and/or after policy implementation as it allows more precise specification of effects-for example, taking into account temporal trends that were occurring before the implementation of the policy. A third strategy is the measurement of policy-specific variables that are theorised to be affected initially after the policy is implemented. For example, in evaluating the impact of a new warning label policy on behaviour, one might reasonably predict that for the policy to exert its effect on behaviour, the target population must first report noticing the new warning labels. ${ }^{12}$ A fourth strategy is the measurement of policy-specific variables for policies that have not changed; such variables act as another form of control. $\overline{\text { For }}$ example, we would expect that in a country where labels have been enhanced and where taxation has not, label salience would be enhanced over time, but taxation-relevant variables (for example, perceived cost of cigarettes) would not.

Combining these four strategies, accompanied by the inclusion of other explanatory variables (covariates) that might help explain differences between two jurisdictions, creates a powerful research design allowing more confident inferences to be made about the causal effects of policies and/ or combinations of policies. ${ }^{3}$ The design and the survey instruments used in the ITC Project together satisfy all of these conditions. ${ }^{16}$ The ITC Project was established with the goal of measuring the psychosocial and behavioural impact of the FCTC policies on (primarily) adult smoking behaviours. ${ }^{16}$ As smokers are most directly affected by tobacco control policies, this understanding is crucial to assessing the extent to which they meet their objectives and of desirable and undesirable collateral effects. The survey component of the ITC Project currently consists of a set of parallel prospective cohort surveys of representative samples of adult smokers in nine countries-Canada, United States, United Kingdom, Australia, Ireland, Thailand, Malaysia, South Korea, and China. The ITC evaluation framework utilises multiple country controls, a longitudinal design, and a pre-specified, theory-driven conceptual model to test hypotheses about the anticipated effects of given policies.

\section{CONCEPTUAL MODEL OF THE ITC PROJECT}

In addition to designing the ITC Surveys to allow us to make strong inferences about the effectiveness of policies, we set out to determine how policies may achieve their desirable effects. For example, we asked whether graphic warning labels have their effect because they merely heighten the attention that smokers pay to the warnings or whether they have their effect because they increase the perceived susceptibility (perceived risk) of the health hazards of smoking.

We developed a conceptual model of how tobacco control policies might work based on a combination of existing 
models from the psychosocial literature and from health communication theories. ${ }^{17-20}$ The resulting conceptual model, which is presented in fig 1 , guided the selection of questions included in all ITC Surveys.

Our conceptual model assumes that each policy ultimately has an influence on behaviour through a specific causal chain of psychological events. Our conceptual model is a general framework for thinking about policies and their effects on a broad array of important psychosocial and behavioural variables, and for testing how policy distinctions relate to their effectiveness. ${ }^{17-20}$

Several key characteristics of this conceptual model require further explanation. First, the model focuses on how policies affect the behaviour of individual smokers, and thus circumvents the vexing problem of making inferences about individuals from aggregates (that is, policy studies in which countries are the unit of analysis, or in individual-level studies that are repeat cross-sectional and for which analyses are conducted over time).

Second, policies are seen as potentially affecting individuals along a variety of psychosocial and behavioural variables, of which there are two classes. The most immediate effects are those on the policy-specific variables - that is, those variables that are proximal (conceptually closest), or most specifically related to the policy itself. Thus, new graphic warning labels should increase salience and noticeability of warnings; price should affect perceived costs of cigarettes (for example, belief that cigarettes have become too expensive); and lifting of restrictions on alternative nicotine products should lead to increased awareness of the availability of those products. These effects may also increase the likelihood of discrete behaviours specifically linked to the manifestations of the policy such as smokers hesitating, or even forgoing or stubbing out cigarettes because of the warning labels. Examples of survey questions designed to measure policyspecific variables are presented in table 2 .
The more downstream effects are on the non-specific psychosocial mediators, which are conceptually distant from the policy and which are theorised to be affected by multiple means, not just policies. Among these are variables such as self-efficacy and intentions, which come from well-known psychosocial models of health behaviour, including the theory of planned behaviour, ${ }^{17}$ social cognitive theory, ${ }^{18}$ the Health Belief Model, ${ }^{19}$ and Protection Motivation Theory. ${ }^{20}$ We believe that policies will affect these general mediating variables indirectly, through their prior effects on the policyspecific variables. As each policy has its own policy-specific variables, to the extent that these are independent, it will allow us to estimate the relative contributions of various policies to the outcomes of interest.

Third, our model explicitly identifies the mediators of policy and articulates the goal of understanding the psychosocial processes that explain how and why a given policy may lead to changes in smoking behaviour. Our longitudinal design allows us to test the causal chain of effects that is depicted in the model; a repeat cross-sectional design would not.

The policy-relevant outcomes that we are measuring include those that confer public health benefits (for example, quitting) but also include important compensatory behaviours that the smoker may engage in that, although responsive to the policy, may not lead to the economic and public health benefits that are ultimately the goal of such policies. For example, smokers may switch to discount brands in response to price increases, which would confer no public health benefit. The ITC Project thus attempts to provide a more complete picture of the effects that may result from the implementation of a tobacco control policy, and this complete picture includes both the detection of desirable effects and of unintended, undesirable side effects.

In summary, the general conceptual model is a causal chain model, and as such, suggests that the policy-specific

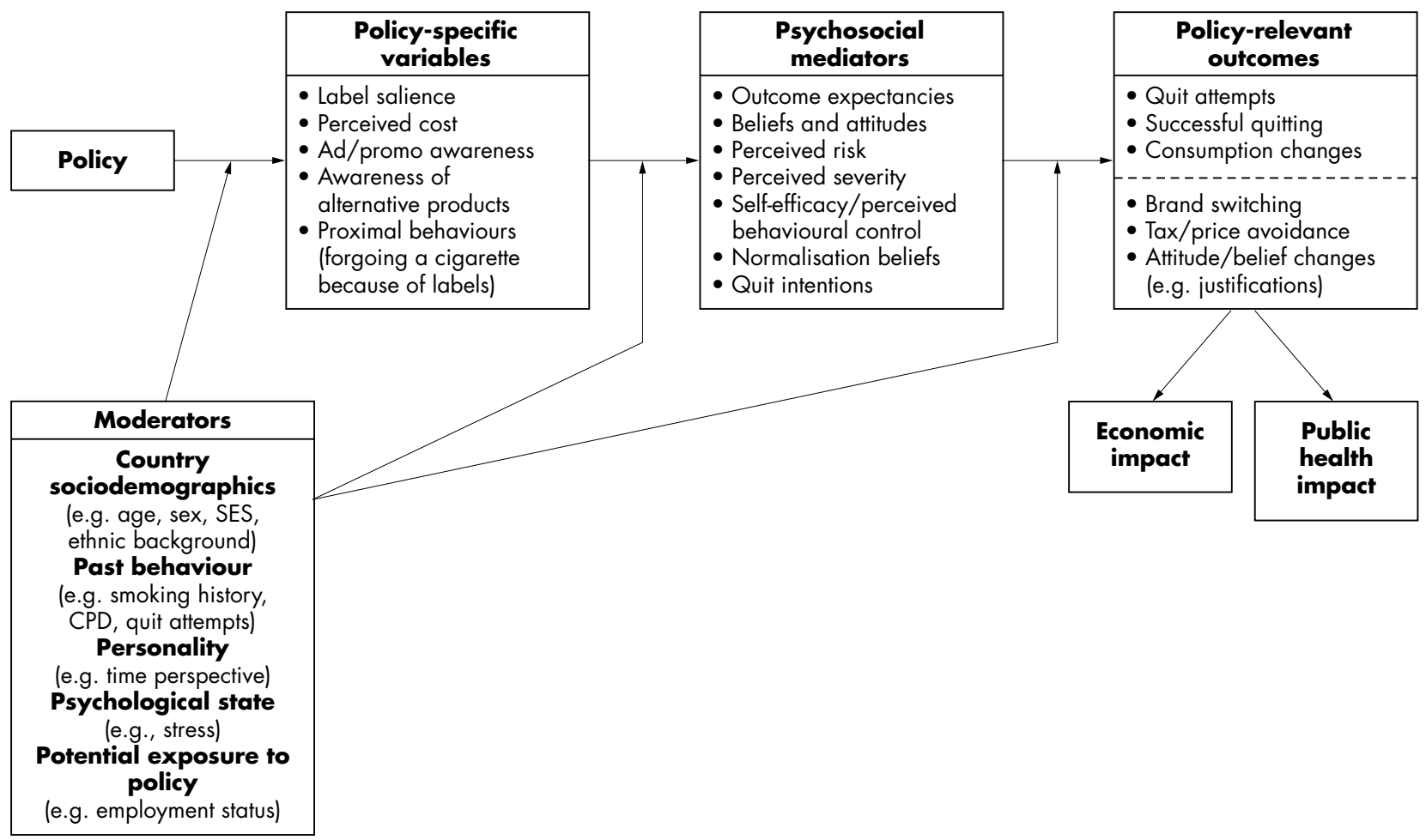

Figure 1 Conceptual model illustrating the hypothesised causal chain of how tobacco control policies exert their influence on tobacco use behaviours. CPD, cigarettes per day; SES, socioeconomic status. 
Table 2 Examples of questions designed to measure policy-specific variables in the International Tobacco Control Surveys

\begin{tabular}{ll}
\hline $\begin{array}{l}\text { Policy } \\
\text { domain }\end{array}$ & Examples of questions measuring \\
policy-specific variables
\end{tabular}

variables play a critical mediating role because they reside between the policy and the outcome variables that are important in public health-for example, quitting behaviour. These causal paths from policy-specific variables to behaviour could be direct, but more typically, will be through the more general mediators, and indeed, in some cases there may be pathways through several kinds of mediators, both policyspecific and non-policy specific. We theorise that policies vary in the psychosocial "routes" that they take to affect behaviour-that is, we have specified different mediational models for each policy domain. For example, noticing warnings may first increase perceived risk of the hazards of smoking, which should affect overall attitudes and outcome expectancies, which affect intentions, which in turn affect behaviour. The design of our study is guided by the possibility of disentangling the web of alternative explanations and competing forces through the careful selection of specific mediators.

Our conceptual model provides us with the opportunity to test how policies impact or fail to impact behaviour as anticipated. For example, the mere existence of a policy, even if implemented properly, does not guarantee that smokers will be exposed to its consequences in the ways anticipated. Taking the example of warning labels again, some smokers barely look at a pack when they are smoking and may rarely or never notice the warnings. This, however, could be due to motivated avoidance, and it is important to measure whether this has an impact on behaviour. In a longitudinal survey of Ontario smokers, Hammond et $a l^{21}$ found that avoidance of the graphic Canadian warning labels by means such as covering them up or by putting them in a cigarette case was not associated at follow-up with a decreased likelihood of a quit attempt. Additional questions can be addressed. For example, is it enough for someone merely to notice warnings, or does one need to read them or otherwise contemplate and accept them? What role do micro-behavioural reactions, such as foregoing a cigarette as a result of noticing/reading, play in determining longer-term outcomes, such as quitting? In order to address these and other conceptual questions about the impact of warning labels, we have included multiple measures to determine, empirically, from our survey results which measures may be important in understanding the impact of warning labels. In this regard, we should note that the "best" measure for understanding the impact of warnings may depend on whether the warning is text-based or whether it includes graphic images. We have recognised the complexities of testing these possibilities at the same time as we recognise their importance and have built in these multiple measures in each policy domain to test these more specific hypotheses about the impact of tobacco control policies.

\section{MODERATOR VARIABLES}

One of the most interesting lines of inquiry in the ITC Project is to determine whether a given policy differs in its impact as a function of country. In the near future, for example, we will be able to determine whether the impact of graphic warnings differs across Thailand, Australia, and the UK, three ITC countries that have either implemented graphic warnings (Thailand), announced that they will (Australia), or is currently considering implementation (UK). Thus country is an important moderator variable in our conceptual model.

Further, within a country, we will be testing for differential policy impact on subgroups of a population, and have thus included variables to determine which subgroups are more favourably (and less favourably) influenced by FCTC policies. These moderators fall into five broad classes: sociodemographics (for example, age, sex, SES, ethnic background); past behaviour (for example, smoking history, current consumption (cigarettes per day), quit attempts); personality characteristics (for example, time perspective, coping style, tendency to avoid or confront potential stressors); other environmental effects (for example, stress levels); and potential exposure to policy (for example, unemployed people will be less affected by workplace smoking policies). In the latter case, we sometimes restrict the analyses to the affected sector of the population. Dealing with hypothesised moderators is relatively straightforward when they are postulated merely to add predictive power to linear models. The issues become more complex when different mediational pathways are postulated for subpopulations. For example, warning avoiders might change behaviour through more emotionrelated pathways, while those who take in the information, might be influenced through more cognitive pathways. The ITC Project has the capacity to build separate models for these different subpopulations and thus to test conceptual models of the health behaviour at the national and international level.

\section{WHAT HAPPENS IF OUR THEORETICAL MODELS ARE WRONG?}

Our goals are to evaluate the impact of policy and to understand the mechanisms of impact. If our mediational 
models are not confirmed in our analyses of policy impact, this provides an opportunity to explore the reasons why and, to the extent possible, to build alternative models that might help us understand why a policy had (or failed to have) its desired impact. One theoretical innovation we have made in the ITC Surveys is to include variables from a range of theoretical frameworks to enable us to test alternative conceptions. ${ }^{17-20}$ For example, in several policy domains, we have included not only the standard strength of belief measures, but also measures of frequency. Although these measures are not conventional in public health, they are of importance in social psychological research, and will allow us to test fine-grained conceptualisations based on attitude accessibility, rather than just the more conventional measure of attitude strength. ${ }^{22}$

\section{WHAT POLICIES ARE WE ABLE TO EVALUATE THROUGH THE ITC SURVEYS?}

ITC Surveys are designed to evaluate the effects of policies on smokers, and increasingly, as our cohorts mature, recent exsmokers. ${ }^{16}$ We are evaluating all of the demand reduction provisions in the FCTC as implemented in the ITC countries: packaging and labelling (Article 11), including health warnings and the elimination of potentially misleading descriptors (for example, "light" or "mild), restrictions/bans on advertising, promotion, and sponsorship (Article 13), protection from exposure to tobacco smoke (Article 8), price and tax measures to reduce demand (Article 6), dependence and cessation (Article 14), and some aspects of education, communication, training, and public awareness of the dangers of tobacco use (Article 12).

We have recently started to assess changes in cigarette brands produced by manufacturers to assess more directly how they have altered their cigarette brands in response to different policies, and to be able to factor these changes in to our understanding of the determinants of changes in smoking patterns. In addition, with the formation of the Roswell Park Transdisciplinary Tobacco Use Research Center (TTURC) funded by the US National Institutes of Health (NIH), we, in collaboration with the US Centers for Disease Control and Prevention, are increasing our capacity to understand cigarette engineering and how that might change in response to policy initiatives, which is the focus of Articles 9 and 10 of the FCTC (see below).

\section{THE ITC FOUR COUNTRY SURVEY}

Based on the above principles, we created the International Tobacco Control Four Country Survey. Table 2 presents examples of policy-relevant variables, psychosocial mediators, and behavioural variables that were included in the ITC Four Country Survey, which is available at: http://www. itcproject.org/Research_Methods/research_methods.htm.

The specific details of our survey methods, along with key survey statistics, are presented in Thompson et al. ${ }^{16}$ The ITC Four Country Survey is a random digit dialled telephone survey of over 2000 randomly selected smokers in each of the four largest English-speaking countries: Canada (where we created a French version of the survey for the francophone regions), the United States, the United Kingdom, and Australia. Wave 1 was conducted during October-December 2002, Wave 2 during May-August 2003, Wave 3 during JuneDecember 2004, and Wave 4 from September-December 2005. Beginning in 2005 with Wave 4, these annual survey waves will be conducted during the last quarter of the year, through 2009. At each wave, cohort members lost to attrition are replaced by newly recruited respondents from the same sampling frame. Thus, at each wave, the ITC Four Country Survey incorporates both a cohort design and a repeat cross-sectional design. This dual design feature allows us to measure the effects of attrition and time-in-sample. ${ }^{16}$

In many areas, these four countries are at the cutting edge of tobacco control policies or initiatives, and since the survey began, and over the next few years, there will be a number of major national-level tobacco control policies implemented in at least one of them. And so, the ITC Four Country Survey will allow us to evaluate the implementation of different FCTC policies at multiple points in time within the same country, with multiple countries as control/comparison groups.

Over time, we will also be able to test the effects of implementations of the same tobacco control policy across different countries. For example, in 2006, Australian warning labels will include graphic photographs. Similarly, following the recent enabling Directive from the European Commission, UK warning labels may also include graphic images in the near future. We will thus be able to compare the impact of similar policies in different countries-and thus to begin addressing issues of the homogeneity versus heterogeneity of policy effects across different countries. Our capacity to do so is enriched owing to the launch of the ITC Southeast Asia Survey in Thailand and Malaysia in January 2005, described below in more detail. Thailand introduced graphic warnings in March 2005, and Malaysia is currently considering doing the same in the future. Because all ITC surveys share identical/similar measures from the identical conceptual framework and analytic plan, we will be able to compare the effectiveness of the graphic warnings in Thailand, Australia, and the UK.

\section{THE UTILITY OF THE ITC CONCEPTUAL MODEL IN THE ITC FOUR COUNTRY SURVEY}

Several of the papers in this supplement illustrate the utility of the model to understanding how tobacco control policies affect distal mediating variables and smoking behaviour indirectly, through their prior effects on the policy-specific variables. ${ }^{12} 24-2628$ Harris et al ${ }^{24}$ examined changes from Wave 1 and Wave 2 of the ITC Four Country Survey on the noticing of advertising/promotion in three different channels: billboards/posters, newspapers and magazines, and sponsorship of sports events. Between Wave 1 and Wave 2, the UK implemented a comprehensive ban on advertising and promotion. Our theoretical model predicted that noticing the presence of tobacco products, advertising, or sponsorship would decline in the UK, relative to the other three countries. Indeed, the findings from Harris et al ${ }^{24}$ shows precisely that pattern as policy-relevant variables changed only in those channels where the policy was changed over time and not in those channels where the policy was left unchanged among the same individuals in the same country. Moreover, whereas Canadian smokers did not vary from the USA and Australia in noticing the presence of tobacco messages in a number of channels, they reported a significant decline in noticing tobacco sponsorships of sporting events, owing to Canada's own imminent ban on tobacco sponsorships coinciding in time with the UK's sponsorship ban. This kind of specificity-precisely as would be predicted by the pattern and timing of advertising/promotion/sponsorship policiesdemonstrates strong convergent and discriminant validity of the ITC Survey.

\section{EVALUATION OF SUB-NATIONAL POLICIES}

We recognise that important tobacco control policies are implemented not only at the national level, but also at subnational levels within a country. Sub-national policies are most apparent in the USA and Canada, with variation in environmental tobacco smoke (ETS) restrictions, taxation, and product regulation at the state and provincial level ${ }^{25-27}$ 
which might be regulated nationally in other countries. Taxation is implemented at a national level in Australia and the UK, as are most other policies in the UK, except ETS restrictions that are currently being developed separately in Scotland and England, and are state-based in Australia. Where the sample sizes are sufficiently high we will test for the effects of sub-national policies.

We have demonstrated this capacity using Wave l data from the ITC Four Country Survey to explore how variation in tobacco taxes and strength of indoor smoking rules impact smoking behaviour and relevant beliefs across different regions of the USA. ${ }^{23}{ }^{25}{ }^{26}$ In New York State, where cigarette prices are among the highest in the USA, 25\% of the New York smokers in our survey reported buying most of their cigarettes from a low or untaxed source such as the internet, compared to less than $2 \%$ in the rest of the USA. ${ }^{23}{ }^{25}$ Those who resided in the seven states that increased cigarette excise taxes between Wave 1 and Wave 2 were more likely to report making special efforts such as travelling to another state to purchase cheaper cigarettes compared to those who lived in states that did not increase excise taxes. ${ }^{23} 25$ A similar subnational analysis of smokers revealed much stronger support for smoking bans in places like California that have already enacted such rules, compared to localities not covered by a ban on indoor smoking. ${ }^{26}$ These data have also provided utility for educating policymakers as they have been presented to help identify the benefits and potential pitfalls of tax increases and clean indoor air policies. It is clear from these two examples that although our focus is on national level policies, we do indeed have the ability to identify and examine differences within-country and disseminate those data to key target audiences.

\section{CURRENT AND FUTURE DIRECTIONS FOR THE ITC PROJECT}

As the ITC Project has evolved and expanded, we have endeavoured to establish a platform and infrastructure for a sustained cooperative research effort with countries throughout the world to evaluate the psychosocial and behavioural effects of tobacco control policies. The ITC Project began its evaluation of FCTC policies in four high-income countries that have been among the leading countries in tobacco control. We did so because of the need to first establish the design and protocols of the overall evaluation effort in countries where the research capacity and resources were available to conduct large-scale national surveys. Demonstrating the viability and power of the ITC Survey design in the four countries has allowed us to move forward toward our goal of adding countries that would vary on important dimensions, notably on economic development, global region, and in countries where tobacco control policies (notably of the FCTC) were likely to be implemented in the near future. Over the past three years, we have thus engaged in strategic expansion so that the present set of ITC participating countries constitute a broader, more diverse set of countries within which to evaluate the policies of the FCTC. Our future plans for expansion will broaden the set of ITC countries to an even greater extent.

We now describe the expansion of the ITC Project beyond the ITC Four Country Survey.

\section{ITC Ireland/UK Survey}

In December 2003, we launched the first wave of the ITC Ireland/UK Survey to evaluate the March 2004 comprehensive workplace smoke-free law in Ireland. ${ }^{28}$ The survey methods were virtually identical to the ITC Four Country Survey, consisting of a random digit dialled telephone cohort survey, with 1000 adult smokers in Ireland, and a parallel control/comparison survey of 600 adult smokers in the UK.
The cohort was followed up during December 2004-January 2005, after the smoke-free law had been in place for about eight months. The ITC Ireland/UK Survey is the first quasiexperimental evaluation study of the effects of the March 2004 comprehensive smoke-free law in Ireland, and demonstrated that the law led to a near-total reduction of tobacco smoke in key public venues such as in bars/pubs, where reported presence of smoking fell from $98 \%$ to $4 \%$ in Ireland in contrast to the UK, where exposure to tobacco smoke remained the same. This was accompanied by significant increases in support for the smoke-free law among our national representative sample of adult smokers in Ireland. These results are presented by Fong et al in this supplement. ${ }^{28}$ In January 2006, we launched an expansion of the ITC Ireland/UK Survey over three years to evaluate the impact of Scotland's smoke-free law (implementation date: 26 March 2006), using England as the control. And England will implement its own comprehensive smoke-free law in 2007, which we will evaluate with this same ongoing cohort survey. In addition to bolstering the size of the cohort in Scotland, we also added a nationally representative cohort of non-smokers in Scotland, England, and the other parts of the UK to enrich our evaluation of the impact of smoke-free policies over time.

\section{ITC Surveys in Asia: Thailand, Malaysia, South Korea, and China}

A second important expansion of the ITC Project is in Asia, where over half of the world's smokers live, and where consumption is expected to increase in the coming decades, particularly among females, who currently are significantly less likely to smoke. Our first expansion was in two middleincome, Southeast Asia countries-Thailand and Malaysia. The ITC Southeast Asia Surveys are parallel cohort surveys of 2000 adult smokers and 1000 youth in each country. We employed a multistage cluster sampling design, with face-toface interviews in the adult smoker sample and selfcompleted questionnaires in the youth sample. The ITC Southeast Asia Surveys were based on the original ITC Four Country Survey in that they included items from each of the demand reduction policy domains of the FCTC, and the question wording was identical or very similar, changing only in cases when pilot testing indicated that an item was not easily understood. The initial wave of the ITC Southeast Asia Survey was conducted January-March 2005, just before the introduction of the graphic warning labels in Thailand in late March 2005. Our current plan is to conduct yearly follow-up survey waves, and at each wave, to employ the same replenishment procedures as in the ITC Four Country Survey-recruitment of new respondents from the same sampling frame.

Thailand and Malaysia serve as models for tobacco control in developing countries within Asia and beyond as both countries have committed to a range of policy initiatives and have an established network of tobacco control researchers and resources. Thailand and Malaysia also have important similarities such as per capita gross domestic product and geographical proximity. There are also important differences between the two countries. Thailand has had a long history of strong tobacco control policies and programmes whereas Malaysia has only recently begun its commitment to strong tobacco control. In addition, religious and cultural differences between the two countries will allow us to examine the relation of these factors to tobacco control policy. In short, Thailand and Malaysia meet both the conceptual and pragmatic requirements for the ITC Project, and the inclusion of both countries will allow us to examine the impact of key tobacco control policies in social, political, and economic environments that vary considerably from those in the original four countries. If we can demonstrate similar effects 
of policies in these countries to those occurring in our original four (affluent, largely English speaking) countries, it will help to demonstrate the generality of policy impact. However, differences in response to policies will allow us to understand what kinds of policy differences are required (if any) in particular cultures.

An important feature of the ITC Southeast Asia Project is the inclusion of the youth survey. As in Western countries, the great majority of smoking initiation in Asia occurs in adolescent populations. The inclusion of a youth cohort of the ITC Southeast Asia Survey allows us to identify the determinants of uptake, the transition to adult smoking, and impact of various policies on this process in Thailand and Malaysia. In addition, it will provide us with the potential for examining similarities and differences in how youth and adults may respond to tobacco control policies in those two countries.

In November 2005, we launched the ITC Korea Survey, a collaboration of the ITC research team and researchers at the National Cancer Center of South Korea. The ITC Korea Survey is a random digit dialled telephone survey of 1000 adult smokers in South Korea. The survey instrument itself, along with the sampling design and protocol, are identical or very similar to the ITC Four Country Survey and ITC Ireland/ UK Survey, which will allow strong comparisons to be made.

In April 2006, we launched the first wave of the ITC China Survey, a collaboration of the ITC research team and the China Centers for Disease Control and Prevention. China is home to $30 \%$ of the world's smokers-a total of about 350 million-and in recognition of the ominous future that these statistics portend, China ratified the FCTC in August 2005. The ITC China Survey will be an annual prospective cohort survey (face-to-face) of about 5600 adult smokers and 1400 adult non-smokers across seven cities in China: Beijing, Shanghai, Guangzhou, Zhengzhou, Changsha, Yinchuan, and Shenyang. The multistage sampling design is similar to the design used in the ITC Southeast Asia Surveys, and the survey instrument, as with all ITC Surveys, consists of core items evaluating all of the FCTC demand reduction policies, with some China-specific measures added to the core survey instrument. In our construction of the sampling frame, we are enumerating 6000 households in each city, and because we are recording smoking status of all household members, we will be able to generate very good estimates of smoking prevalence in each city. Indeed, the ITC China Survey is being used by the China Office of Tobacco Control as a tool for China's surveillance efforts in the context of the FCTC. Wave 1 of the ITC China Survey launched in April 2006.

Conducting the ITC Survey in these four Asian countries will allow comparisons to be made within Asia along a number of important dimensions-for example, comparisons between high versus middle/low income countries (Korea versus China, Thailand, Malaysia), and history of tobacco control policies (Thailand being the country with the longest history versus the other three countries, with more recent commitments to tobacco control). All four countries have ratified the FCTC, and will begin (or continue) to implement FCTC policies in the near future. With our multiple country design, within Asia, and in the other non-Asian countries, the ITC Project is positioned to identify the commonalities as well as the dissimilarities of the impact of FCTC policies in this critical region of the world, and to make comparisons in policy impact between Asian and non-Asian countries of the ITC Project.

\section{Further expansion and addressing the issue of generality versus specificity}

As our expansion continues (at this time, we are developing ITC Surveys in New Zealand, France, Mexico, and Uruguay, with additional efforts toward developing similar projects in India and in other countries), we will begin to address the important basic question of whether the effectiveness of a given tobacco control policy is relatively consistent or inconsistent in its effectiveness across income levels, across cultures, and across geographical regions. If policy effectiveness varies across countries, we may be able to identify factors that may explain that variability. At a broader level, these questions about generality versus specificity of policy effects across countries is part of a broader set of important questions about the extent to which one country's experiences with tobacco (or tobacco control, or other health challenges) can generalise to those of another country.

An initial foray into addressing this broader issue of generality versus specificity was made by Fong et al, ${ }^{29}$ who examined the level of regret among smokers in the ITC Four Country Survey. We found that the experience of regret about smoking ("if you had to do it over again, you would not have started smoking") was nearly universal (about 90\%) and no different across the four countries. Moreover, the predictors of regret (a long and broad list, including demographic variables, perceived addiction, perceived cost, anticipated future health damage from smoking) did not vary in their predictive power across the four countries: the country $x$ predictor interaction was not statistically significant, despite the very high statistical power available because of the very large sample size. Whatever cultural differences may exist across these four countries, the experience of regret and the factors that predict regret are no different. It remains to be seen whether the level of regret and the factors that predict regret varies across the broader range of countries that have joined the ITC Project. An initial investigation by Lee et $a l^{30}$ of ITC Southeast Asia Survey data has shown that the level of regret in Thailand is similar to that of the original four countries, but that the level of regret in Malaysia is significantly lower (about $80 \%$ ). The difference may reflect the fact that Thailand has had a longer history of strong tobacco control policies and advocacy, with norms for tobacco use being considerably more negative than in Malaysia, which has only more recently made a strong commitment to tobacco control.

As the ITC Project goes forward in our expansion process, we will be able to address the question of generality versus specificity, commonality versus uniqueness, in the experience of tobacco use, and, importantly for the trajectory of the FCTC, in the impact of FCTC policies.

\section{ITC PRODUCT TRACKING PROJECT}

It is becoming increasingly clear that the tobacco problem requires a coherent and integrated systemic response that takes into account both the motivations and understandings of smokers, but also the motivations and actions of those who market tobacco products. ${ }^{31-34}$ Evidence from internal industry documents reveals that cigarette manufacturers have been successful in large measure by their ability to adapt to a changing policy environment. ${ }^{31}$ For example, cigarette manufacturers changed their marketing strategies in response to government efforts to warn consumers about the health risks of smoking (for example, creation and increased promotion of low tar brand extensions) and restrict advertising (for example, event sponsorships, direct marketing through mailing lists). It is important for policy researchers to not only study how policies impact consumers but also to learn how the industry adapts its products and marketing efforts to maximise sales or otherwise maintain their profitability.

Towards this goal, the ITC Project has established the first international repository of selected popular cigarette brands as a way of monitoring and evaluating changes in cigarette 
brands in participating ITC countries. The ITC Product Tracking Project will assess how cigarette design and smoke chemistries change in relationship to policies, and how product modifications alter smokers' perceptions, behaviours (brand choice, puff topography), and exposures (as measured by key biomarkers). The overall mission of the ITC Product Tracking Project is to build the scientific foundation for regulatory and policy activity that is called for in the FCTC (Articles 9 and 10). As important as it is to create evidencebased policies, it is perhaps most critical for us to do so in the domain of product regulation, and to do so in the international context. Recent initiatives to revise the existing protocols for measuring emissions (the International Standards Organisation/Federal Trade Commission (ISO/ FTC) protocol) has highlighted our need to engage in research that will inform such revision-a process that is currently dominated by the tobacco industry. ${ }^{34}$

\section{LIMITATIONS OF THE ITC PROJECT}

The ITC Project does not have the tools or capacity to do all that is needed to evaluate effectively all aspects of the FCTC. We are not evaluating "the FCTC". We are evaluating the impact of FCTC policies as implemented by a growing set of countries that vary on important dimensions-size, economic development, geographic region, culture, and prior history of tobacco control. This will allow us to begin to address issues of generality versus specificity of FCTC policy impact across countries.

Second, apart from our youth surveys in Malaysia and Thailand, we are not examining the effects of tobacco control policies on youth uptake. This will require the GYTS, particularly if complemented by ITC-like cohort surveys of youth.

Third, we are somewhat limited in our ability to examine the impact of FCTC policies on non-smokers, except in Scotland and England, where we have added a non-smoker cohort to gain a more complete understanding of the impact of comprehensive smoke-free policies, and also to a limited extent in Malaysia and China.

Fourth, we cannot evaluate the direct effects of policies that affect the behaviour of tobacco companies via our surveys; rather, we are limited to measuring the consequences that may be known about or experienced by smokers. In these cases, however, ITC data may be valuable when used in conjunction with data from other sources. For example, understanding smuggling and other forms of excise tax avoidance might make use of ITC price data and data on atypical packaging, but this endeavour will need to be complemented by other forms of enquiry, such as Malaysia's recent introduction of data matrix codes on cigarette packaging.

Fifth, our ITC product studies focus on cigarettes, cigarette smoke, and cigarette smokers, thus representing a first step toward the goal of evaluating the role of product differences in tobacco use. At present, we are not conducting ITC studies in countries with high levels of smokeless tobacco use; however, we are keen to work with other countries with high levels of smokeless tobacco use (for example, India) to extend our evaluation efforts in these areas.

Sixth, our conceptual model focuses on how a single policy influences behaviour, rather than on how combinations of policies interact to influence behaviour. However, our evaluation efforts include countries in which more than one FCTC policy has been implemented, and thus our analytic modelling of the impact of policies will allow us to provide at least some initial indications about the impact of multiple policies (and the possibility of interactions among policies) than exists today. In fact, the ITC Project has the potential to empirically test interactions among policies,

\section{What this paper adds}

The Framework Convention on Tobacco Control (FCTC), the first-ever international treaty on health, is a seminal event in tobacco control and in global health. Over 120 countries have ratified the FCTC, but whether the FCTC eventually results in significant reductions in the harms of tobacco use will depend on the strength of the implementation of FCTC policies among the ratifying countries. Evidence that supports FCTC policies is needed, particularly from international cohort studies.

The International Tobacco Control Policy Evaluation Project (ITC Project) consists of a set of prospective cohort surveys of representative samples of adult smokers in nine countries so far, inhabited by over $45 \%$ of the world's smokers-Canada, United States, United Kingdom, Australia, Ireland, Thailand, Malaysia, South Korea and China. Youth cohorts are being surveyed in Thailand and Malaysia, and non-smoker cohorts are being surveyed in the United Kingdom, Malaysia, and China. Together, the ITC Project Surveys constitute the firstever international cohort study of tobacco use. The objectives of the ITC Project are to evaluate the psychosocial and behavioural impact of all of the demand reduction policies of the FCTC, to understand how FCTC policies have their impact on individuals, and to test whether policy impact varies as a function of country and other moderating variables (for example, sociodemographic variables, personality characteristics, past behaviour of individual smokers). This article describes the conceptual framework underlying the ITC Project, which includes specifications of mediational models that trace the route(s) from policy to behavioural outcomes (for example, quitting) through policy-specific variables and general psychosocial mediators. The ITC Project provides a framework and method that could be utilised to evaluate and understand the impact of policies in other domains of public health.

owing to the commonality of methods, study design, and survey instrument, across different countries. As our understanding of individual policies increases, and if we find evidence of interactions between policies, we will extend our theorising to try to understand these as well, but that is for the future.

\section{CONCLUSION}

The FCTC represents an extraordinary landmark in global tobacco control, but the path from the promise of effective tobacco control policies at the global level to the reality of strong implementation of FCTC policies will not be easy. Many countries have not yet ratified the FCTC, and in many countries that have already ratified the FCTC, there is pressure either to delay the implementation of the FCTC or to implement FCTC policies in ways that will render them less effective than their potential. The mission of the ITC Project is to conduct rigorous evaluation of FCTC policies in order to establish the evidence base that will give policymakers throughout the world the evidence base that will facilitate ratification in those countries that have not yet ratified the FCTC, and strong implementation in those countries that have ratified. ITC Project findings presented in this supplement ${ }^{12}{ }^{24-26} 28$ are an initial collection of findings from the ITC Project that support the FCTC's potential as an instrument for reducing the harms of tobacco use. At the same time, these findings illustrate how our conceptual model can be applied to understanding how tobacco control policies may ultimately exert their influence on downstream psychosocial variables such as knowledge, beliefs, attitudes 
and intentions, and on subsequent tobacco use behaviours. Identifying the mechanisms through which tobacco control policies operate will provide tools for reducing the harms of tobacco use that could be employed even in the absence of changes in policies, or to increase the effectiveness of policies that are being implemented.

The positive accelerating trajectory of the toll of tobacco use in the 21 st century represents a major threat to global health, one that demands a mobilisation and alignment of researchers, advocates, and governments toward meeting the threat. We hope that through the efforts of our ITC Project team and those of other tobacco control researchers throughout the world, the FCTC process will fulfil its promise-global implementation of effective tobacco control policies, informed by evidence from the best available research.

\section{ACKNOWLEDGEMENTS}

We thank Craig Steger and Christian Boudreau for their comments on preliminary versions of this article. We thank Taryn Sendzik, Tara Elton-Marshall, Alexandra Lin Marie Fong, Stacey Jahn, Carol Murray, Ruth Loewen, and Pete Driezen for their assistance.

\section{Authors' affiliations}

G T Fong, Department of Psychology, University of Waterloo, Waterloo, Ontario, Canada

K M Cummings, A Hyland, G A Giovino, Department of Health Behavior, Roswell Park Cancer Institute, Buffalo, New York, USA R Borland, Cancer Control Research Institute, The Cancer Council Victoria, Carlton, Victoria, Australia

G Hastings, Institute for Social Marketing and Centre for Tobacco Control Research, University of Stirling and the Open University, Stirling, UK

D Hammond, Department of Health Studies and Gerontology, University of Waterloo, Waterloo, Ontario, Canada

M E Thompson, Department of Statistics and Actuarial Sciences, University of Waterloo, Waterloo, Ontario, Canada

The ITC Project is supported by grants R01 CA 100362 and P50 CA111236 (Roswell Park Transdisciplinary Tobacco Use Research Center) from the National Cancer Institute of the United States, Robert Wood Johnson Foundation (045734), Canadian Institutes of Health Research (57897), National Health and Medical Research Council of Australia (265903), Cancer Research UK (C312/A3726), Canadian Tobacco Control Research Initiative (014578); Centre for Behavioural Research and Program Evaluation, National Cancer Institute of Canada/ Canadian Cancer Society. Role of the funding sources: The funding sources had no role in the study design, in the collection, analysis, and interpretation of data, in the writing of the report, and in the decision to submit the paper for publication.

Competing interest statement: All authors declare that the answer to the questions on the competing interest form www.bmj.com/cgi/content/ full/317/7154/291/DC1 are all "No" and therefore have nothing to declare.

Ethics approval: This manuscript is a review and presentation of the conceptual framework of the International Tobacco Control Policy Evaluation Project and as such, there are no primary data presented in this manuscript. Hence, ethics approval is not relevant for this manuscript.

\section{REFERENCES}

1 Ezzati M, Lopez AD. Estimates of global mortality attributable to smoking in 2000. Lancet 2003:362:847-852.

2 World Health Organization. Framework Convention on Tobacco Control Geneva, WHO, 2003 (http://www.who.int/tobacco/framework/download/ en/ (Accessed 17 December 2004).

3 Framework Convention on Tobacco Control. http://www.fctc.org/ index.php.

4 Cook TD, Campbell DT. Quasi-experimentation: design and analysis issues for field settings. Boston: Houghton Mifflin, 1979
5 Chaloupka FJ, Hu TW, Warner KE, et al. The taxation of tobacco products. In: Jha $P$, Chaloupka F, eds. Tobacco control in developing countries. New York: Oxford University Press, Inc, 2001:237-72.

6 Borland R. Tobacco health warnings and smoking-related cognitions and behaviours. Addiction 1997;92:1427-35.

7 Borland R, Hill D. Initial impact of the new Australian tobacco health warnings on knowledge and beliefs. Tob Control 1997;6:317-25.

8 The COMMIT Research Group. Community Intervention Trial for Smoking Cessation (COMMIT): I. Cohort results from a four-year community intervention. Am J Public Health 1995;85:183-92.

9 Hyland A, Li Q, Baver JE, et al. Effect of state and community tobacco control programs on smoking cessation rates in adult smokers. Am J Health Prom 2005:29:85-90.

10 Wakefield M, Chaloupka F. Effectiveness of comprehensive tobacco control programmes in reducing teenage smoking in the USA. Tob Control 2000;9:177-86.

11 Farrelly MC, Pechacek TF, Chaloupka FJ. The impact of tobacco control program expenditures on aggregate cigarette sales: 1981-2000. J Health Econ 2003;22:843-9.

12 Hammond D, Fong GT, McNeill A, et al. Effectiveness of cigarette warning labels in informing smokers about the risks of smoking: findings from the International Tobacco Control (ITC) Four Country Survey. Tob Control 2006;15(suppl III):iii19-25.

13 Fong GT. Evaluating the Effects of the September 2003 European Union Policy Banning "Light/Mild" Cigarette Brand Descriptors: Findings from the International Tobacco Control Policy Evaluation Survey. Report Submitted to Health Canada, January 13, 2005 (Contract \#, 4500095771).

14 Velicer WF, DiClemente CC. Understanding and intervening with the total population of smokers. Tob Control 1993;2:95-6.

15 Warren CW, Riley L, Asma S, ef al. Tobacco use by youth: a surveillance report from the Global Youth Tobacco Survey project. Bull Wrld Health Organ 2000;78:868-76.

16 Thompson ME, Fong GT, Hammond D, et al. Methods of the International Tobacco Control (ITC) Four Country Survey. Tob Control 2006;15(suppl III):iii 12-18.

17 Ajzen I. The theory of planned behavior. Organizational Behavior and Human Decision Processes 1991;50:179-211.

18 Bandura A. Social foundations of thought and action: a social-cognitive theory. Englewood Cliffs, New Jersey: Prentice-Hall, 1986.

19 Becker MH. The health belief model and personal health behavior. Thorofare, New Jersey: Charles B Slack, 1974.

20 Rogers R, Prentice-Dunn S. Protection motivation theory. In: Gochman D, eds. Handbook of health behavior research: Vol. 1. Determinants of health behavior: personal and social. New York: Plenum, 1997:113-32.

21 Hammond D, Fong GT, McDonald PW, et al. Impact of the graphic Canadian warning labels on adult smoking behaviour. Tob Control 2003;12:391-5.

22 Fazio RH, Williams CJ. Attitude accessibility as a moderator of the attitude perception and attitude-behavior relations: an investigation of the 1984 presidential election. J Personality and Soc Psych 1986;51:505-14.

23 Cummings KM, Hyland A, Travers $M$, et al. Cigarette purchase patterns and cigarette prices: US Data from the International Tobacco Control Policy Evaluation Survey. Paper presented at the Society for Research on Nicotine and Tobacco, Arizona, February, 2004

24 Harris F, Mackintosh AM, Anderson S, et al. Effects of the 2003 advertising/ promotion ban in the United Kingdom on awareness of tobacco marketing: findings from the International Tobacco Control (ITC) Four Country Survey. Tob Control 2006;15(suppl III):iii26-33.

25 Hyland A, Laux FL, Higbee C, et al. Cigarette purchase patterns in four countries and the relationship with cessation: findings from the International Tobacco Control (ITC) Four Country Survey. Tob Control 2006; 15(suppl III):iii59-64.

26 Borland R, Young $\mathrm{H}-\mathrm{H}$, Siahpush $\mathrm{M}$, et al. Support for and reported compliance with smoke-free restaurants and bars by smokers in four countries: findings from the International Tobacco Control (ITC) Four Country Survey. Tob Control 2006;15(suppl III):iii34-41.

27 O'Connor RJ, Giovino GA, Fix BV, Hyland A, et al. Smokers' reactions to reduced ignition propensity cigarettes. Tob Control 2006;15:45-9.

28 Fong GT, Hyland A, Borland R, et al. Reductions in tobacco smoke pollution and increases in support for smoke-free public places following the implementation of comprehensive smoke-free workplace legislation in the Republic of Ireland: findings from the ITC Ireland/UK Survey. Tob Control 2006;15(suppl III):iii51-8.

29 Fong GT, Hammond D, Laux FL, et al. The near-universal experience of regret among smokers in four countries: findings from the International Tobacco Control Policy Evaluation Survey. Nic Tob Res 2004;6(suppl 3):S341-51.

30 Lee WB, Zanna MP, Fong GT, et al. Regret, rationalization, and intentions to quit among smokers in Malaysia and Thailand: findings from the ITC Southeast Asia survey. Paper to be presented at the 13th World Conference on Tobacco or Health, Washington, DC. (July 2006).

31 Pollay RW, Dewhirst T. The dark side of marketing seemingly light cigarettes: successful images and failed fact. Tob Control 2002;11:18-31.

32 Gray N. Reflections on the saga of tar content: why did we measure the wrong thing? Tob Control 2000;9:90-4.

33 Wu W, Zhang L, Jain RB, et al. Determination of carcinogenic tobacco-specific nitrosamines in mainstream smoke from U.S. brand and non-U.S. brand cigarettes from 14 countries. Nicotine Tob Res 2005;7:443-51.

$34 \mathrm{O}^{\prime}$ Connor RJ, McNeill A, Cummings KM, et al. How did UK cigarette makers reduce tar to $10 \mathrm{mg}$ or less? BMJ 2006;332:302. 